\title{
A Curriculum on Naval Science \& Technology for a Midwestern University
}

\section{Dr. James Buchholz, University of Iowa}

James Buchholz is an Associate Professor of Mechanical Engineering at the University of Iowa. He received the Bachelors and Masters degrees in Mechanical Engineering from the University of Alberta, and the Ph.D. degree in Mechanical and Aerospace Engineering from Princeton University. He teaches courses in fluid mechanics and conducts research in unsteady aerodynamics and hydrodynamics.

\section{Dr. Jae-Eun Russell, University of Iowa}

Dr. Russell serves as the Director of Research \& Analytics Office of Teaching, Learning \& Technology. Dr. Russell conducts learning analytics research to learn about how to promote the adoption of datainformed instructional practices that support successful student learning. Her research is focused on student learning, engagement and motivation, facilitated by teaching practices, and technology adoptions in various learning environments.

\section{Venanzio Cichella, University of Iowa}

Prof. Venanzio Cichella received his B.S. and M.S. in Automation Engineering in 2007 and 2011, respectively, from the University of Bologna, Italy. He got his Ph.D. in Mechanical Engineering in 2018 from the University of Illinois at Urbana-Champaign, majoring in planning and control of multiple autonomous systems. He is currently an Assistant Professor at the Mechanical Engineering department at the University of Iowa. His research interests include cooperative control of autonomous systems, collision avoidance, optimal control, machine learning, and human-centered autonomous vehicle design.

\section{Prof. Casey Harwood, University of Iowa}

Casey Harwood is an assistant professor of Mechanical Engineering and an affiliate of IIHR - Hydroscience and Engineering at the University of Iowa. He received his B.S. in Naval Architecture \& Marine Engineering from the Webb institute, and his M.S. and Ph.D. in the same from the University of Michigan. He teaches courses in thermo-fluid dynamics and experimental methods. His research group focuses on experiments in nonlinear marine dynamics, fluid-structure interactions, and multi-phase flows.

\section{Dr. Shaoping Xiao, University of Iowa}

Shaoping Xiao is an associate professor of Mechanical Engineering and an affiliate of ITI - Iowa Technology Institute (formerly CCAD) at the University of Iowa. He received his B.S. and M.S. in Mechanics from the University of Science and Technology of China, and his Ph.D. in Mechanical Engineering from Northwestern University. He teaches courses in machine learning, artificial intelligence, and Nonlinear Control. His research group focuses on computational material science, multiscale modeling, and robotics.

\section{Dr. Pablo M. Carrica, University of Iowa}

P. M. Carrica is a professor with the Department of Mechanical and Industrial Engineering at The University of Iowa. He teaches courses in the area of fluid mechanics, and is an active researcher in hydrodynamics of surface and underwater vehicles, working on bubbly wakes, cavitation and maneuvering and seakeeping. His research team develops the computational naval hydrodynamics code REX. 


\section{A Curriculum on Naval Science \& Technology for a Midwestern University}

\section{Introduction}

From a national defense perspective, there is strong demand for quality educational programs that can prepare students to pursue careers in naval science \& technology (Kiss, 2011; Lundquist, 2014). Because there are relatively few institutions in the United States that offer comprehensive naval architecture programs, there is an opportunity for smaller programs to help meet the need by developing quality curricula on Navy-related S\&T subjects, and to make students aware of potential careers in this area.

The program discussed in this paper has been developed at the University of Iowa, which provides unique challenges for attracting students with interest in naval science $\&$ technology. It is therefore important to cultivate that interest through exposure to relevant curricular topics and engineering challenges. In addition, due to the limited faculty resources, the curriculum must be developed without substantial expansion in the number of courses offered.

To address the national need, we developed an undergraduate certificate in naval hydrodynamics (Buchholz et al., 2018), which students can earn through their selection of elective courses in the baccalaureate degree. Whereas the program has achieved success in attracting students and placing them in Navy labs and industry contractors, graduates at this level of academic preparation must be prepared to deal with a broad range of challenges. It is therefore beneficial to increase the breadth of the naval curriculum. We have recently strategically expanded the certificate, within our resource constraints and expertise, to also include courses in autonomous systems and control. The new certificate is titled, "Naval Science \& Technology". To augment the curriculum and provide opportunities for a greater number of students to participate in naval science \& technology challenges, we also work closely with an extracurricular student club that is strongly aligned with the curriculum.

This paper describes the structure and content of the curriculum and student organization, illustrating their synergies. We also analyze student interest in naval science \& technology challenges, the certificate program, and careers in the Navy and its contractors using survey data collected in undergraduate seminars.

\section{Program structure}

The Certificate in Naval Science \& Technology can, in principle, be earned by any undergraduate student at the institution, regardless of academic discipline; however, so far, participants have been exclusively mechanical engineering students. The restriction on certificate participants is primarily due to course prerequisites, which include courses in fluid mechanics, differential equations, dynamics, and control. It is anticipated that the program will ultimately attract some students in other disciplines of engineering; however, the curricula of 
other engineering disciplines typically does not include all of the needed prerequisites, so that students would need to take extra courses.

To broaden participation in the subject, as well as to support students in certificate courses, a student organization has been established, which is named the Iowa Marine Autonomous Racing Club (IMARC), which addresses technical challenges that are strongly complementary to the curriculum. Presently, the primary focus of IMARC is to design and build an autonomous boat, and compete in the RoboBoat competition (https://roboboat.org) sponsored by the Office of Naval Research (ONR) and the Association of Unmanned Vehicle Systems International (AUVSI). Specifically, there is strong correspondence between the curriculum and the RoboBoat challenges, as they both teach students autonomous systems concepts including motion planning, navigation, and control; and naval hydrodynamics concepts including propulsion, resistance, and maneuvering.

Having a student organization that is closely aligned with the curriculum provides opportunities for students to become involved in naval science \& technology who may not otherwise have the opportunity due to course prerequisites or competing degree objectives. We are also exploring its use as a somewhat formal pedagogical tool since the student organization can support curricular learning by providing realistic and challenging applications in which students can further develop their curricular knowledge and skill. Similarly, courses can implement assignments and projects that emulate RoboBoat challenges to foster the development of critical technical skills within the student organization.

The certificate consists of 18 credits (6 courses), according to the formula described in Table 1 . Required elements of a student's plan of study include courses in naval hydrodynamics, control and autonomous systems, and an approved capstone project on a naval science \& technology topic, which can be either a full-year senior design elective (which also satisfies the ABET capstone design experience requirement) or a single-semester independent investigation with a faculty advisor.

\section{Curriculum and facilities}

The primary certificate courses are listed in Table 1, and are arranged into the categories of naval hydrodynamics courses, control \& autonomous systems courses, and capstone courses. Course learning objectives focus on technical knowledge associated with the subject as well as current naval science \& technology challenges and career opportunities. Informing students on the latter topics is done, in part, through presentations by Navy and contractor staff. Each of the major courses in the curriculum, and their corresponding infrastructure is summarized below. 
Table 1: Naval Science \& Technology Certificate overview

\begin{tabular}{|l|}
\hline Naval hydrodynamics courses (students must complete at least two) \\
- ME:4125 Biomimetic Fluid Dynamics \\
- ME:4175 Computational Naval Hydrodynamics \\
- ME:4176 Experimental Naval Hydrodynamics \\
Autonomous Systems and Machine Learning courses (students must complete at least two) \\
- ME:4111 Scientific Computing and Machine Learning \\
- ME:5114 Nonlinear Control in Robotic Systems \\
- ME:5115 Cooperative Autonomous Systems \\
Capstone course (students must complete at least one): \\
- Full-year capstone design elective on a naval S\&T topic \\
- one semester of supervised independent study on an approved naval S\&T topic \\
General electives \\
- Any additional courses needed to complete the 18 credit requirement can be selected \\
from a list of approved courses on topics including control, robotics, artificial \\
intelligence, fluid mechanics, materials, and manufacturing.
\end{tabular}

\section{Naval hydrodynamics courses}

The Mechanical Engineering program at the University of Iowa is of relatively small size, with twenty tenure-system and instructional faculty, providing limited capacity to expand the number of naval hydrodynamics courses. They must therefore be attractive to a broad section of students in order to attain a sustainable enrollment. In order address the resource and enrollment constraints, the courses are built around the more general themes of computational methods (ME:4175), experimental methods (ME:4176), and the fluid dynamics of bio-inspired vehicles (ME:4125). Each of these courses requires an introductory fluid mechanics course as a prerequisite, and is offered every second year, with their schedules staggered such that at least one of the courses is offered each year.

In ME:4175, simulations based on relevant vessels (DTMB 5512) and propellers (KP505) are used to introduce the use of computational fluid dynamics for the analysis of surface and underwater marine craft performance, while also introducing naval hydrodynamics concepts related to resistance, propulsion, maneuvering and seakeeping. The propulsion unit includes simulations of a propeller open water curve and description of how a self-propulsion computation produces propulsion factors (thrust deduction, Taylor wake fraction, rotative and propulsive efficiency, etc.). Seakeeping response is studied by performing pitch and heave simulations in head waves, while maneuvering includes a static (pure drift) simulation and computation of maneuvering derivatives. A complete example is worked in class using the generic submarine DARPA SUBOFF advancing in irregular waves.

ME:4175 has been taught in the past using a teaching version of the in-house naval hydrodynamics software REX. REX is an advanced code with considerable capabilities to treat a large number of hydrodynamics problems related to surface and underwater vehicles, and has been successfully used in the class and also by students in capstone design projects. However, it suffers from two main drawbacks: 1) it requires licenses for the grid generation software 
(Gridgen), the overset domain connectivity information (Suggar++) and for post-processing (Tecplot) and 2) REX is proprietary software and cannot be distributed to the students after they leave the University. These drawbacks limit the extent of the use the students can make of their learning in computational fluid dynamics in their careers. To provide the students with tools that can be used freely, ME:4175 is switching to OpenFOAM as CFD software. While OpenFOAM lacks many of the capabilities that REX has for naval hydrodynamics problems, it is still very capable and is an open-source code that includes grid generation (snappyHexMesh) and postprocessing (paraFoam) tools with a free GNU General Public License, so students can continue working with OpenFOAM after graduation. Transition from REX to OpenFOAM is accompanied by a full set of online tutorials for remote delivery of the course.

ME:4176 Experimental Naval Hydrodynamics equips students with the laboratory and analytical skills necessary to conduct fundamental experimental testing of naval systems. Students who complete the course can conduct resistance testing of hulls, perform open-water testing of propellers, make inertial measurements of models, and measure the linear seakeeping responses of ship models in regular waves. All these skills are both fundamental to a naval experimentalist and highly specialized. We instruct students through lecture and hands-on experience with research-grade equipment in the Advanced Measurements Laboratory. Students have access to an instructional towing tank, which is equipped with a measurement carriage, resistance dynamometer, and a wave-maker. Recent upgrades include a gimballed mount that permits students to record heave, pitch, and roll motions of a towed model, as well as a precision swing table that students use to record and adjust the mass distribution of models.

In addition to standard naval architecture tests, students also learn the fundamentals of common instruments, perform instrument calibrations, learn data acquisition topics like quantization and data rates, and use this knowledge to rigorously quantify uncertainty in their own experiments. Each student also proposes, designs, and conducts their own experiment to address a research question of naval relevance. Figure 1 shows a collection of video stills captured by students who proposed to study the process by which air is ingested into highly-loaded propellers operating at shallow depths. The group of students systematically varied the immersion depth and revolution rate of the propeller and used triggered acquisition of high-speed video from multiple angles to track the ingestion of air, which was later compared with time-resolved measurements of the thrust and torque to demonstrate that air ingestion caused a severe drop in the efficiency of the propeller. 


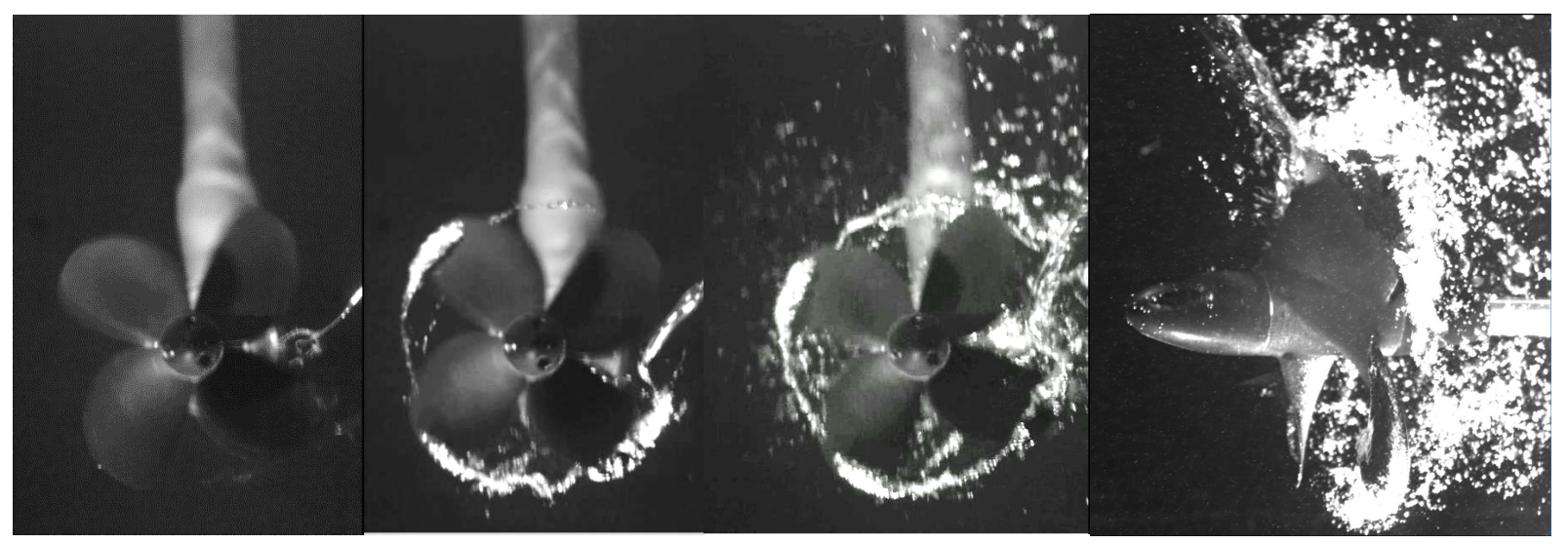

Figure 1: High-speed video still from a student project that sought to measure the extent and effects of air entrainment or "ventilation" on shallowly-submerged propellers.

ME:4125 Biomimetic Fluid Dynamics addresses the unsteady hydro/aerodynamics associated with biological and bio-inspired swimming and flight. Shallow-water dynamic environments, such as within the surf zone, and regions of complex sea-floor topography, present significant challenges for low-speed maneuvering and station keeping, in which marine animals excel. In addition, biological swimmers exhibit advantages over traditional marine propulsion systems in the areas of stealth and maneuverability, and potentially also propulsive efficiency (Anderson et al., 1998; Fish and Lauder, 2006). The course focuses on the basic physics underlying locomotion in fluids, while enriching the students' experiences and ensuring naval relevance through collaboration with scientists and engineers at the Naval Research Laboratory in a major project. Students study the application of dimensional analysis, conservation laws, aerodynamic theory, and models of unsteady thrust and lift production to canonical problems of biological locomotion in fluids.

Students interact extensively with engineers at the Naval Research Laboratory who introduce the students to naval science \& technology challenges in bio-inspired locomotion, and mentor students in a major course project of current naval relevance. The project addresses the design and performance of bio-inspired fins for maneuvering in dynamic flow environments. A servomotor-driven flapping-fin apparatus is used to articulate fins in a low-turbulence visualization water channel that is well-suited for dye visualization, and optically-based measurement methods such as laser Doppler anemometry, particle image/tracking velocimetry, and image-based methods for tracking fin deformation. The flow-field measurements complement measurements of fin thrust, angular displacement/velocity, and shaft torque in order to help students integrate their developing knowledge of conservation laws and vortex dynamics into the projects. Figure 2 provides an example of a project in which students leveraged a recent study published by the NRL group (Ramamurti et al., 2019) to design and experimentally test fin designs. In the final project, each team addresses a particular facet of the problem, such that the breadth of their understanding is expanded presentations 

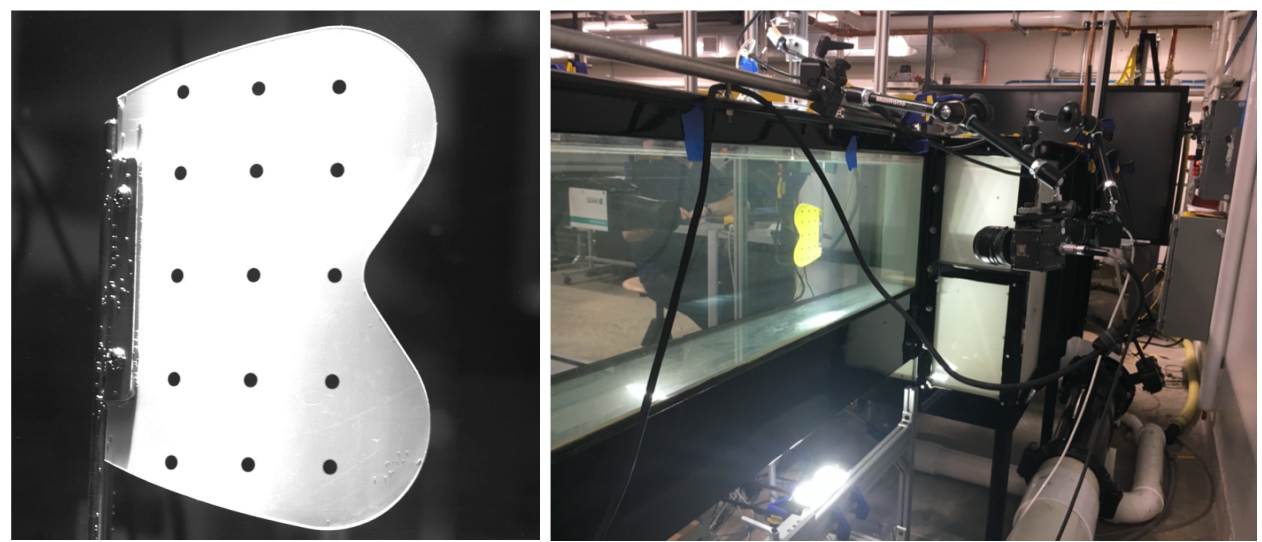

Figure 2: Investigation of a flexible fin modeled after a zebra danio. (left): The fin model with dots printed for optical tracking of fin deformation during the oscillation cycle. (right): The fin operating in a water channel, imaged with high-speed cameras to quantify fin deformation.
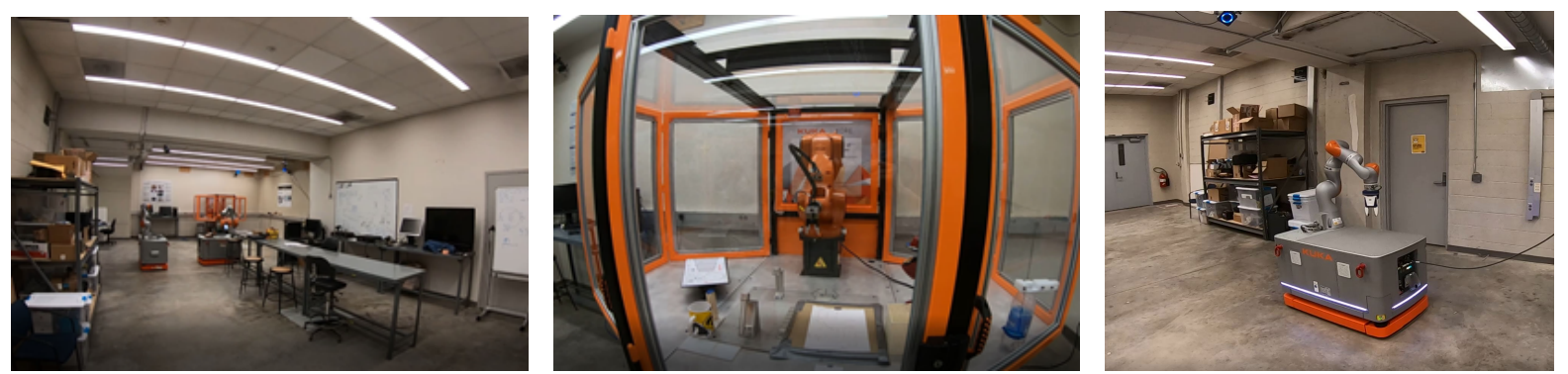

Figure 3: Control, Automation, and Robotics (CAR) lab with KUKA robots. The "Control, Automation and Robotics" (CAR) lab in the Department of Mechanical Engineering supports departmental teaching, including ME:5114. The CAR lab is equipped with one KR C4 robotics manipulator and two KUKA KMR (KUKA Mobile Robotics) iiwa omniMoves with robot arms. The lab also has an OptiTrack Prime Camera system, which enables high-speed tracking with excellent precision in medium-sized capture volumes.

\section{Control and autonomous systems courses}

The control and autonomous systems courses align with a departmental focus in this area. Projects are conducted within the Control Automation and Robotics laboratory shown in Figure 3, where students can program and track ground robots using state-of-the-art image-based localization technology. Future course-related projects will also involve underwater robotic vehicles.

ME:4111 Scientific Computing and Machine Learning was revised two years ago, focusing on the computational and data-driven approaches for engineering problem-solving. The course objective is to introduce students to scientific computing and machine learning methods that are commonly used in science and engineering computations. This course is designed for sophomore and junior students, as the first engineering elective course that they can take. Therefore, it serves as a general and foundational course for the certificate program. In addition to mechanical engineering students, students from other engineering departments (e.g., Civil, Biomedical) and other colleges (e.g., Liberal and Arts, Business) are also interested in this course, which may 
provide a means of introducing a broader range of students to naval S\&T challenges in the future. The emphasis is on developing an understanding of appropriate scientific computing algorithms and machine learning models, and mastering problem-solving skills using numerical methods and machine learning software packages. Algorithms are implemented in Python. This course covers the following major topics: solution of root and optimization problems, solution of systems of linear and nonlinear equations, numerical differentiation and integration, solution of ordinary and partial differential equations, and data science process via machine learning. Students are required to participate in team projects to conduct data analytics via various machine learning models. Students participating in the robotic boat team are encouraged and advised in integrating course concepts into their autonomous boat analyses and algorithms.

ME:5114 Nonlinear Control in Robotics Systems is one of the advanced control courses offered to mechanical engineering junior and senior undergraduate students. This course is intended to expose students to nonlinear analysis and control systems theory. The course is focused on nonlinear autonomous and nonautonomous systems as well as the applications of nonlinear control design tools on robotics systems. Lyapunov-based analysis methods and associated design techniques are emphasized. The first segment of the course introduces definitions of stability for autonomous and nonautonomous systems leading to a Lyapunov framework. Then, based on the developed Lyapunov-based stability analysis tools, basic and advanced design tools for contemporary engineering problems are presented including state-of-the-art techniques. Python programming is utilized in this course. Also, some supplementary topics are introduced, including advanced control techniques (LQR, MPC, Kalman filter), reinforcement learning, and data-driven control, which . Students will conduct open-ended team projects to apply the control design tools to robotic systems. Projects related to autonomous boats are incorporated to support the activities of the student organization.

ME:5115 - Cooperative Autonomous Systems is a course designed for senior undergraduate and first-year graduate students on guidance, navigation, and control of multiple autonomous systems. By taking this course, the students gain insight into how to enable ground, marine and aerial robotic platforms to perform cooperative tasks autonomously in complex real-world environments. The theoretical topics that are addressed include optimal control, numerical methods, and nonlinear analysis and control. These tools are exploited to solve problems relevant to autonomous systems, namely trajectory generation, tracking, collision avoidance, and multivehicle coordination. The class uses project-based activities in a laboratory environment, focusing on the design and implementation of algorithms for autonomous system operations. Students gain experience using MATLAB and Robot Operating System (ROS) for advanced control and optimization problems. Course modules enable students to get hands-on experience in algorithm design, implementation, and testing. Students interact with engineers at the Naval Research Laboratory who introduce the students to naval science \& technology challenges in controls and autonomy. This course also supports the student organization by integrating topics relevant to their activities and providing an opportunity for algorithms development which can be used by the student organization. In that regard, course projects are tailored to directly apply to RoboBoat competitions in order to help students to translate their knowledge to relevant problems in naval science and technology that are of interest to the student organization. 


\section{Capstone courses}

For most students, the capstone requirement is met by completing the capstone design elective. Students complete a project of naval relevance in teams. As the naval S\&T section of the senior design course is run in parallel with non-naval sections, the teams can consist of combinations of students who are completing the naval S\&T certificate and those who are not. It is also typical that the teams comprise students with a variety of curricular experiences, with at least some students having previously completed or are concurrently completing the certificate requirements.

Projects vary considerably, usually comprising elements of the IMARC boat or vehicle applications that are broadly of interest to the Navy. In contrast to typical industry-sponsored senior design projects, national security restrictions prohibit students from addressing specific projects of current interest to the Navy and its contractors. However, robust relationships developed with Navy labs through program alumni and graduate research sponsorships have fostered a strong cohort of Navy mentors which provide substantial support to the student teams, who share expertise beyond that of the program faculty, and bring an important Navy perspective to the students' work. Weekly design reviews are conducted with naval S\&T faculty, Navy engineers and, when appropriate, student organization members, providing feedback and technical support to the student teams.

Recent projects have included design of a ducted propeller for the student organization competition boat, a semi-autonomous underwater glider, and elements of an autonomous underwater research submarine model. Figure 4 shows a simulation visualization and prototype model for a propeller used to compete in the Roboboat competition.
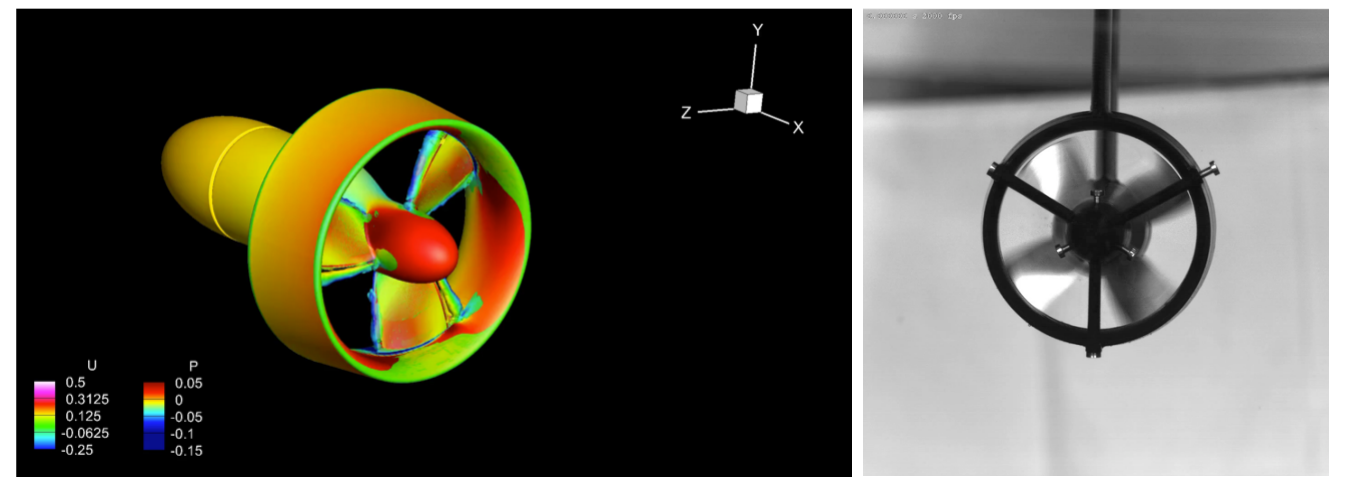

Figure 4: An example naval S\&T capstone design project in which a student team designed a ducted propeller for use on the student organization competition boat. A design iteration is simulated on the left, and the propeller used in competition is shown on the right.

\section{The Student Organization (IMARC)}

In contrast to certificate registration, which is limited to a relatively small number of students (typically 2 to 4 graduates per year) and individual certificate courses, which are primarily taken by mechanical engineering students, participation in the student organization is much more 
inclusive, and is based solely on interest. There is broad representation across the college of engineering, computer science, and other departments within the university. The student team is divided into three technical subgroups: hull and propulsion, software, and hardware. Where possible, technical challenges faced by the hull and propulsion group are addressed in projects within the naval hydrodynamics courses, and those faced by the hardware and software groups are addressed in the control and autonomous systems courses.

The benefits of the close alignment between the curriculum and the student organization are anticipated to extend to both elements of the program, and future investigation is planned (through surveys and focus groups) to better elucidate the relationship. In addition to the curricular support of the student team, naval S\&T faculty contribute through faculty advising of club operations, giving presentations on topics of relevance to the team, and participating in comprehensive design reviews.

\section{Student Perspectives on the Curriculum, Student Organization, and Navy-related Careers}

A survey was conducted to assess student interest in the Naval Science and Technology certificate program and careers in support of the Navy. Participants were students enrolled in the Mechanical Engineering undergraduate seminars in the sophomore and junior years. The survey included questions regarding interest in the Navy and naval science careers, extra-curricular activities, family involvement in the Navy, and overall interest in the certificate program. A total of 88 students participated in the study. 41 students were in the sophomore seminar and 47 students were in the junior seminar. These students were mostly male ( $84 \%$ of the sample), and $17 \%$ of the students were first-generation students. Only five students were underrepresented minorities. Despite that the seminars target students in the sophomore and junior years, students from all four years of the undergraduate program were included in the survey; however most of the sample was second (43\%) and third year (35\%) students. A majority of students were majoring in mechanical engineering, with a few majoring in industrial, civil, and electrical Engineering. The survey followed presentations by faculty on the Naval Science \& Technology certificate, and by a Navy recruiting officer on naval S\&T challenges.

A large majority of participants showed interest in the science and technology challenges faced by the Navy and its industry contractors ( $85 \%$ overall). Despite this, less than half of participants reported interest in pursuing a Navy related career ( $42 \%$ overall). Fewer participants displayed interest in pursuing the certificate program (33\% overall). These percentages were similar across both seminars. Thus, naval science and technology challenges are of interest; however, other factors prevent that interest from translating to a similar interest in Naval careers specifically or the Naval Science \& Technology certificate program.

Given the broad nature of the mechanical engineering discipline, and the lack of local employment opportunities in naval applications, the number of students interested in Navyrelated careers and the certificate program are considered to be very high. However, it is noteworthy that the actual number of graduates who have completed the certificate has typically ranged between approximately 2 and 4 out of a graduating class size of around 100, with similar numbers of students continuing in graduate study with naval focus, or obtaining employment 
with the Navy or its contractors. Therefore, interest is clearly not translating to action, so there appears to be an opportunity to significantly increase certificate enrollment with targeted advising or other strategies to help these students transition.

Most participants reported that they had sufficient understanding of how to pursue career paths in the Navy and its industry contractors (77\% overall). This item, however, yielded response differences across the two seminars, such that the students from the junior seminar reported greater understanding (85\%) than ones from the sophomore Seminar (68\%). This difference makes sense considering that the junior seminar consists of mostly third and fourth year students, who are much more likely to have completed a naval S\&T course, or attended additional presentations by Navy or naval industry speakers, while the sophomore seminar is mostly comprised of second year students who are just becoming acquainted with mechanical engineering after completing a common freshman year.

There was no difference across gender when assessing student understanding of different kinds of Naval careers and how to pursue them; however, females were less likely to be interested in these careers overall (23\% for females compared to the $42 \%$ overall). Differences also emerged among first-generation students, who reported higher interest in Navy-related careers, but less sufficient understanding to make informed decisions about pursuing those kinds of careers. There were no major differences noted between under-represented minorities and other students, although the number of under-represented minorities in the sample is too small to draw strong conclusions.

When survey results were filtered according to students' academic year of study, third year students reported the most interest in the certificate program. $48 \%$ of third year students were interested in the certificate program. Fourth year students, on the other hand, reported the least interest across academic year. This is a likely a reflection of the fact that fourth-year students who have not already begun to complete the certificate will not have an opportunity to do so without delaying graduation. Although there was no difference regarding interest in the program for males, females on average were less likely to be interested (23\%). Additionally, firstgeneration students on average were more interested in the program (47\%).

Participants were also asked to indicate their interest in joining IMARC. Overall, 44\% reported interest in joining the organization and some of them requested to be contacted to learn more about the club. Interestingly, students who indicated interest in joining also reported more interest in pursuing naval careers than the students who indicated no interest in the club $59 \%$ of those with interest in joining vs. $29 \%$ of those without). Likewise, students who indicated interest in joining IMARC also reported more interest in the certificate program than the ones who showed no interest in IMARC (56\% of those with interest in joining vs. $14 \%$ of those without).

\section{Summary and Conclusions}

This paper documents a new program established at the University of Iowa to teach engineering principles related to naval science \& technology, and to facilitate students joining the workforce 
in support of the Navy. The program consists of a curricular undergraduate certificate program, focusing on naval hydrodynamics and autonomous naval systems, as well as a student organization (IMARC) focused on developing autonomous boats that compete in the international Roboboat competition. The goals of the curriculum and the student organization are well-aligned, providing mutual benefits that will be explored in further detail in future studies. Specifically, the curriculum and faculty participation in the student organization activities provides strong technical background to support the activities of the club, whereas the student organization broadens the pool of students who are able to participate in naval science and technology, expanding the reach of the program. Despite that the students at the University of Iowa are predominantly from midwestern states, and come to the university with limited experience in naval systems, there is a high level of interest in naval science \& technology challenges, which translates to moderate degrees of interest in careers in support of the Navy, and in the curricular program. This suggests that small programs such as the one described in this paper have the potential to make a substantial impact on workforce development in naval science \& technology.

\section{References}

Anderson J.M., Streitlien, K., Barrett, D.S., and Triantafyllou, M.S. (1998). Oscillating foils of high propulsive efficiency, J. Fluid Mech. 360, 41-72.

Buchholz J., Carrica P., and Russell, J.-E. (2018). A naval hydrodynamics undergraduate curriculum for the midwesten United States, ASEE Annual Conference, Salt Lake City, UT. 24 27 June, 2018. Paper ID 22450.

Fish, F.E. and Lauder, G. V. (2006). Passive and active flow control by swimming fishes and mammals, Annu. Rev. Fluid Mech. 38, 193-224.

Kiss, R.K. (2011). Examining the science and technology enterprise in naval engineering workforce and education, Special Report 306, Committee on Naval Engineering in the $21^{\text {st }}$ Century, Transportation Research Board.

Lundquist, E. (2014). Navy, industry must replace experienced workforce; learn how to achieve stability and predictability, Naval Engineers Journal, 126(3), pp. 37-43.

Ramamurti R., Geder, J.D., Viswanath, K., and Pruessner, M. (2019). Propulsion characteristics of flapping caudal fins and its upstream interaction with pectoral fins. AIAA SciTech Forum, 711 January, 2019, San Diego, CA. 